\title{
Changes in postmastectomy radiotherapy targets in breast cancer in China between 1999 and 2008: an epidemiological study
}

\author{
Shu-Lian Wang • Ye-Xiong Li • Bao-Ning Zhang • Qing-Kun Song • \\ Jing Li • Jin-Hu Fan • Bin Zhang • Hong-Jian Yang • Xiao-Ming Xie • \\ Zhong-Hua Tang • Hui Li • Jia-Yuan Li • Jian-Jun He • You-Lin Qiao
}

Received: 7 May 2013 / Accepted: 5 June 2013 / Published online: 20 June 2013

(C) Springer-Verlag Berlin Heidelberg 2013

\begin{abstract}
Objective To provide evidence that could improve management of breast cancer, we conducted a multi-center retrospective epidemiological study to investigate the status of and changes in postmastectomy radiotherapy (PMRT) targets in mainland China.

Authors' contributions SLW helped to design the CRF, collect, analyze, and interpret the data. She also drafted the initial manuscript. BNZ helped to design the study and is the clinical PI of the study. JHF and QKS helped with the data management and performed the statistical analysis. BZ HJY, XMX, ZHT, HL, JYL, and JJH were the local PIs that helped with data collection. YLQ was the PI of this study and assisted in designing the study. YXL helped to designed the study and performed critical revisions of the manuscript. All authors read and approved the final manuscript.
\end{abstract}

\section{S.-L. Wang $\cdot$ Y.-X. Li $(\bowtie)$}

Department of Radiation Oncology, Cancer Hospital (Institute),

Chinese Academy of Medical Sciences, Peking Union Medical

College, 17 South Panjiayuan Lane, Beijing 100021, China

e-mail: yexiong@yahoo.com

\section{B.-N. Zhang}

Department of breast surgery, Cancer Hospital (Institute), Chinese Academy of Medical Sciences, Peking Union Medical College,

17 South Panjiayuan Lane, Beijing 100021, China

\section{Q.-K. Song $\cdot$ J. Li $\cdot$ J.-H. Fan $\cdot$ Y.-L. Qiao}

Department of Cancer Epidemiology, Cancer Hospital (Institute), Chinese Academy of Medical Sciences, Peking Union Medical

College, 17 South Panjiayuan Lane, Beijing 100021, China

\section{B. Zhang}

Department of Breast Surgery, Liaoning Cancer Hospital, No. 44 Xiaoyanhe Road, Dadong District, Shenyang 110042, China

\section{H.-J. Yang}

Department of Breast Surgery, Zhejiang Cancer Hospital, No. 38 Banshanqiao Guanji Road, Hangzhou 310022, China
Methods Patients who had received PMRT for breast cancer between 1999 and 2008 were randomly selected from seven hospitals. Information on radiation targets, including the chest wall $(\mathrm{CW})$, the supraclavicular fossa (SCF), the axilla, and the internal mammary chain (IMC) was collected and analyzed. The Cochran-Armitage

\section{X.-M. Xie}

Department of Breast Oncology, Sun Yat-Sen University Cancer Center, 651 Dongfeng East, Guangzhou 510060, China

\section{Z.-H. Tang}

Department of Breast-thyroid Surgery, Xiangya Sencod Hospital, Central South University, No. 139 Renminzhong Road, Changsha 410011, China

H. Li

Department of Breast Surgery, the Second People's Hospital of Sichuan Province, Chengdu 610041, China

\section{J.-Y. Li}

Department of Epidemiology, West China School of Public Health, Sichuan University, Chengdu Sichuan 610041, China

\section{J.-J. He}

Department of Oncosurgery, the First Affiliated Hospital of Medical College, Xi' an JiaoTong University, 277 Yanta West Road, Xi'an 710061, China 
trend test and the chi-square test were used to compare differences.

Results Of 661 included patients, 596 (90.2\%), 606 (91.5\%) 196 (29.6\%), and 297 (44.9 \%) had received CW, SCF, axillary, and IMC radiotherapy (RT), respectively. From 1999 to 2008, there was an increase in the use of CW RT $(p<0.001)$, and a decrease in axillary $(p=0.027)$ and IMC $(p<0.001)$ RT. There were significant regional differences in the use of $\mathrm{CW}(p=0.008)$, axillary $(p<0.001)$, and IMC $(p<0.001)$ RT. There was no significant change in the use of SCF RT from 1999 to 2008 ( $p=0.597)$, or between different regions $(p=0.371)$.

Conclusions Variations in postmastectomy radiotherapy targets existed between different Chinese regions during the period studied. The CW and SCF targets were irradiated in most patients. The use of axillary and IMC RT decreased significantly.

Keywords Breast neoplasm · Epidemiological study · Postmastectomy $\cdot$ Radiotherapy $\cdot$ Target

\begin{tabular}{ll}
\multicolumn{2}{l}{ Abbreviations } \\
PMRT & Postmastectomy radiotherapy \\
RT & Radiotherapy \\
CW & Chest wall \\
SCF & Supraclavicular fossa \\
IMC & Internal mammary chain
\end{tabular}

\section{Introduction}

Postmastectomy radiotherapy (PMRT) has been shown to improve locoregional tumor control and overall survival in patients with node-positive breast cancer [1]. In randomized trials, comprehensive loco-regional radiotherapy (RT) is delivered to the chest wall $(\mathrm{CW})$, the axilla, the supraclavicular fossa (SCF), and the internal mammary nodes (IMC) [2-4]. However, results from several large studies evaluating patterns of locoregional failure in patients received adjuvant systemic therapy without RT indicated that the $\mathrm{CW}$ followed by the SCF are the most common sites of locoregional recurrence. Recurrences were less frequent in the axilla and rare in the IMC [5-8]. The results from Eastern Cooperative Oncology Group (ECOG) trials demonstrated that patients with positive axillary lymph nodes commonly developed recurrence in the $\mathrm{CW}$ $(12 \%)$, the supra/infraclavicular nodes $(8 \%)$, the axilla (4\%), and the IMC (0.2\%) [5]. Therefore, the key target for $\mathrm{RT}$ is the $\mathrm{CW}$ and the supra/infraclavicular nodes.

In mainland China, total mastectomy and axillary dissection has been the main surgical approach for most patients with non-metastatic breast cancer regardless of the stage. The appropriate application of PMRT is important to ensure the quality of patient care. However, in a country such as mainland
China with a large and populous geography and limited resources, there is a wide variation in the practice of locoregional radiotherapy for breast cancer. In order to provide evidence that could improve management, we conducted a retrospective epidemiological study to investigate the status of and changes in PMRT targets in the management of breast cancer in seven representative hospitals in China between 1999 and 2008. This study was approved by the institutional review board (IRB) of the Cancer Foundation of China.

\section{Methods}

Patient selection

Mainland China is divided into seven regions (north, northeast, central, south, east, northwest, and southwest), and one tertiary academic hospital from each region was selected. Each hospital has its own RT facilities. Further details on these hospitals can be seen in Table 1 . Patients were randomly selected and the relevant information was collected. One month per year (except for January or February) was randomly selected to represent each year from 1999 to 2008 in every hospital. All inpatient cases within the selected month were reviewed and the information was collected. For each selected month, if patient numbers were fewer than 50 , then more cases from the neighboring months were reviewed until the total number reached 50. If patient number in the selected month exceeded 50, all cases in that month were reviewed. All patients met the following inclusion criteria: (1) pathology confirmed primary breast cancer; (2) the inpatient admission date was within the selected month in the study hospital; and (3) treatment (surgery, chemotherapy, or radiotherapy) for breast cancer was recorded. The method used has been previously described, among 45,200 breast cancer patients treated in seven hospitals during 10 years, 4,211 (9.3\%) patients were randomly selected [9]. Patients who met the following criteria were enrolled in this study: (1) a mastectomy and axillary dissection had been performed; (2) adjuvant postmastectomy radiotherapy had been delivered; and (3) radiotherapy sites were known. A total of 661 patients were included in this study, which accounts for $1.5 \%$ of the total number of breast cancer patients treated in these seven hospitals during 10 years. Table 1 indicated that the number of patients included in this study are not evenly distributed between all the hospitals mainly because of the variations in the use of PMR between hospitals. As the majority of patients in hospital \#3 were treated on out-patient basis, only seven inpatients were included.

Patients were classified into three groups according to disease stage: high-risk group, defined as T3-4, or with four or more positive axillary lymph nodes or positive surgical margins; intermediate-risk group, defined as tumor size $5 \mathrm{~cm}$ or less, with 
Table 1 Information on all seven hospitals (1, north; 2 , northeast; 3 , central; 4 , south; 5 , east; 6 , northwest; and 7, southwest)

\begin{tabular}{lllll}
\hline Hospital & Hospital size (beds) & $\begin{array}{l}\text { Cancer vs. general } \\
\text { hospitals }\end{array}$ & $\begin{array}{l}\text { Total number of breast } \\
\text { cancer patients treated } \\
\text { between 1999 and 2008 }\end{array}$ & $\begin{array}{l}\text { Number of patients } \\
\text { included in the analysis }\end{array}$ \\
\hline 1 & 1,200 & Cancer hospital & 9,536 & 94 \\
2 & 1,500 & Cancer hospital & 11,670 & 132 \\
3 & 2,850 & General hospital & 3,127 & 7 \\
4 & 1,080 & Cancer hospital & 5,637 & 89 \\
5 & 1,370 & Cancer hospital & 8,745 & 160 \\
6 & 2,340 & General hospital & 3,126 & 83 \\
7 & 1,200 & Cancer hospital & 3,359 & 96 \\
\hline
\end{tabular}

one to three positive axillary nodes; and low-risk group, defined as tumor size $5 \mathrm{~cm}$ or less, with negative axillary nodes.

\section{Statistical analyses}

The Cochran-Armitage trend test was used to evaluate trends in the use of different $\mathrm{RT}$ targets and in variations in patient groups from 1999 to 2008. The chi-square test was used to compare differences in the use of RT targets between different hospitals and between different patient groups. Statistical significance was assessed using two-tailed tests with an alpha level of 0.05 . The SAS statistical software was used for data analysis.

\section{Results}

Of 661 patients included, $532(80.5 \%)$ had undergone a modified radical mastectomy, $126(19.1 \%)$ had undergone a radical mastectomy and three patients $(0.4 \%)$ had had an extended radical mastectomy. The median number of axillary nodes dissected was 16 (range, 1-66). Other than the 18 patients $(1.8 \%)$ for whom complete pathology results were not available, the axillary nodes were negative in 127 patients $(19.2 \%)$, one to three nodes were positive in 168 patients $(25.4 \%)$, and four or more were positive in 354 patients $(53.6 \%)$.

The distribution of RT targets for the 661 patients is shown in Table 2. Overall, the $\mathrm{CW}$ was irradiated in 596 patients $(90.2 \%)$, the SCF in 606 patients $(91.5 \%)$, the axilla in 196 patients $(29.6 \%$ ), and the IMC in 297 patients (44.9\%). The RT techniques and the doses used for the $\mathrm{CW}$ and the nodal regions are shown in Table 3 . The majority of patients received a total dose of $50 \mathrm{~Gy}$, using conventional fractionation of 2 Gy per day. Low-energy electrons are used in about $60 \%$ of patients for CW RT and bolus was used in $27.2 \%$ of patients. Combined photon and electron, or photon alone were used frequently for SCF RT.

From 1999 to 2008, there was an increase in the use of CW RT, and a decrease in the use of axillary and IMC RT (Fig. 1). The use of CW RT increased from $67.3 \%$ in 1999 to
$98.4 \%$ in 2003 , then remained relatively stable between 2004 and $2008(p<0.001)$. The use of axillary RT fluctuated between 25.5 and $44.9 \%$ from 1999 to 2005 , then steadily decreased from $36.8 \%$ in 2006 to $22.8 \%$ in 2008 ( $p=0.027$ ). The use of IMC RT decreased significantly from $81.8 \%$ in 1999 to $25.3 \%$ in $2008(p<0.001)$. However, there was no significant change in the use of SCF RT from 1999 to 2008, varying between 83.7 and $96.5 \%$ ( $p=0.597$; Fig. 1$)$.

There were significant differences between different regions in the use of CW, axillary, and IMC RT (Fig. 2). The use of CW RT varied between 71.4 and $97.9 \%$ $(p=0.008)$. The use of axillary RT varied between 3.1 and $85.4 \%(p<0.001)$. The use of IMC RT varied between 14.3 and $85.6 \%(p<0.001)$. However, there was no significant difference between different regions in the use of SCF RT, which varied between 84.1 and $97.9 \%$ ( $p=0.371$; Fig. 2).

Among the 661 patients, 568 patients had available staging information and were divided into low-, intermediate-,

Table 2 Distribution of radiation targets in 661 patients with breast cancer

\begin{tabular}{ll}
\hline Radiation targets & Frequency (percentage) \\
\hline $\mathrm{CW}+\mathrm{SCF}$ & $227(34.3)$ \\
$\mathrm{CW}+\mathrm{SCF}+\mathrm{IMC}$ & $156(23.6)$ \\
$\mathrm{CW}+\mathrm{SCF}+$ axilla & $97(14.7)$ \\
$\mathrm{CW}+\mathrm{SCF}+$ axilla+IMC & $72(10.9)$ \\
$\mathrm{SCF}+\mathrm{IMC}$ & $38(5.7)$ \\
$\mathrm{CW}$ & $28(4.2)$ \\
$\mathrm{SCF}+$ axilla+IMC & $11(1.7)$ \\
$\mathrm{IMC}$ & $10(1.5)$ \\
$\mathrm{CW}+$ axilla+IMC & $6(0.9)$ \\
$\mathrm{CW}+$ axilla & $6(0.9)$ \\
$\mathrm{CW}+\mathrm{IMC}$ & $4(0.6)$ \\
$\mathrm{SCF}+$ axilla & $3(0.5)$ \\
SCF & $2(0.3)$ \\
Axilla & $1(0.2)$ \\
\hline
\end{tabular}

$C W$ chest wall, $S C F$ supraclavicular fossa, IMC internal mammary chain 
Table 3 Radiation techniques and doses to chest wall and nodal regions
$C W$ chest wall, $S C F$ supraclavicular fossa, $I M C$ internal mammary chain

\begin{tabular}{|c|c|c|c|c|}
\hline & CW (\%) & $\operatorname{SCF}(\%)$ & Axilla (\%) & IMC (\%) \\
\hline \multicolumn{5}{|l|}{ Beam } \\
\hline Electron & $362(60.7)$ & $41(6.8)$ & $19(9.7)$ & $68(22.9)$ \\
\hline Photon & $60(10.1)$ & $133(21.9)$ & $54(27.6)$ & $13(4.4)$ \\
\hline Cobalt & $4(0.7)$ & $55(9.1)$ & $50(25.5)$ & $11(3.7)$ \\
\hline Photon+electron & $72(12.1)$ & $189(31.2)$ & $7(3.6)$ & $54(18.2)$ \\
\hline Cobalt + electron & $5(0.8)$ & $92(15.2)$ & $4(2.0)$ & $97(32.7)$ \\
\hline Unknown & $93(15.6)$ & $96(15.8)$ & $148(32.6)$ & $54(18.1)$ \\
\hline \multicolumn{5}{|c|}{ Electron energy for $\mathrm{CW}$} \\
\hline 6 & $355(59.6)$ & & & \\
\hline 8 & $37(6.2)$ & & & \\
\hline 9 & $13(2.2)$ & & & \\
\hline 10 & $17(2.9)$ & & & \\
\hline 12 & $6(1.0)$ & & & \\
\hline Mixed & $11(1.8)$ & & & \\
\hline Unknown & $157(26.3)$ & & & \\
\hline \multicolumn{5}{|c|}{ Dose per fraction (cGy) } \\
\hline 1.8 & $1(0.2)$ & $0(0)$ & $1(0.5)$ & $1(0.3)$ \\
\hline 2.0 & $536(89.9)$ & $548(90.4)$ & $157(80.1)$ & $266(89.6)$ \\
\hline 2.5 & $1(0.92$ & $1(0.2)$ & $0(0$ & $3(0.8)$ \\
\hline 2.9 & $5(0.8)$ & $5(0.8)$ & $0(0$ & $0(0)$ \\
\hline Unknown & $53(8.9)$ & $52(8.6)$ & $38(19.4)$ & $1(0.3)$ \\
\hline \multicolumn{5}{|l|}{ Total dose (cGy) } \\
\hline $1,000-4,000$ & $18(3.0)$ & $19(3.1)$ & $9(4.6)$ & $23(7.8)$ \\
\hline $4,001-4,999$ & $46(7.7)$ & $29(4.8)$ & $7(3.6)$ & $14(4.7)$ \\
\hline 5,000 & $468(78.5)$ & 484 (79.9) & $131(66.8)$ & $224(75.4)$ \\
\hline $5,001-6,000$ & $13(2.2)$ & $22(3.6)$ & $10(5.1)$ & $9(3.0)$ \\
\hline $6,001-7,000$ & $5(0.9)$ & $5(0.8)$ & $4(2.0)$ & $2(0.7)$ \\
\hline Unknown & $46(7.7)$ & $47(7.8)$ & 35 (17.9) & $25(8.4)$ \\
\hline \multicolumn{5}{|l|}{ Bolus for CW } \\
\hline Yes & $162(27.2)$ & & & \\
\hline No & $301(50.5)$ & & & \\
\hline Unknown & $133(22.3)$ & & & \\
\hline
\end{tabular}

and high-risk groups. There were no significant changes in the distribution of low-, intermediate-, and high-risk groups from 1999 to 2008 ( $p=0.768$; Fig. 3). The association between RT targets and risk groups were analyzed for these 568 patients (Table 4). Patients in high-risk groups received CW RT more frequently than low- and intermediate-risk groups. Patients in high- and intermediate-risk groups received SCF RT more frequently than low-risk groups. There was no association between risk group and axillary RT, or between risk group and IMC RT.

The correlation between the use of regional node RT and axillary node status was shown in Table 5. Except for that, the use of axillary RT was associated with positive axillary nodal ratio of $20 \%$ or more. Overall, $23.4 \%$ of patients with a nodal ratio less than 20 and $33.3 \%$ of patients with a nodal ratio $20 \%$ or more received axillary RT $(p=0.007)$. The use of IMC RT was associated with primary tumor location, with $53.8 \%$ of patients with tumor located at the inner quadrant and $42.6 \%$ of patients with tumor at the outer quadrant receiving IMC RT $(p=0.015)$.

\section{Discussion}

In this retrospective epidemiological study, we found that the $\mathrm{CW}$ and the SCF were the two principal targets most often irradiated in PMRT, and more than $90 \%$ of patients had received $\mathrm{CW}$ and $\mathrm{SCF}$ RT. The use of $\mathrm{CW}$ RT increased, and axillary and IMC RT decreased from 1999 to 2008. This finding is consistent with the evidence, which suggests that the $\mathrm{CW}$ and the SCF are the two most common sites of locoregional recurrence, reflecting the American Society of 
Fig. 1 Use of locoregional postmastectomy radiotherapy in patients with breast cancer, from 1999 to 2008

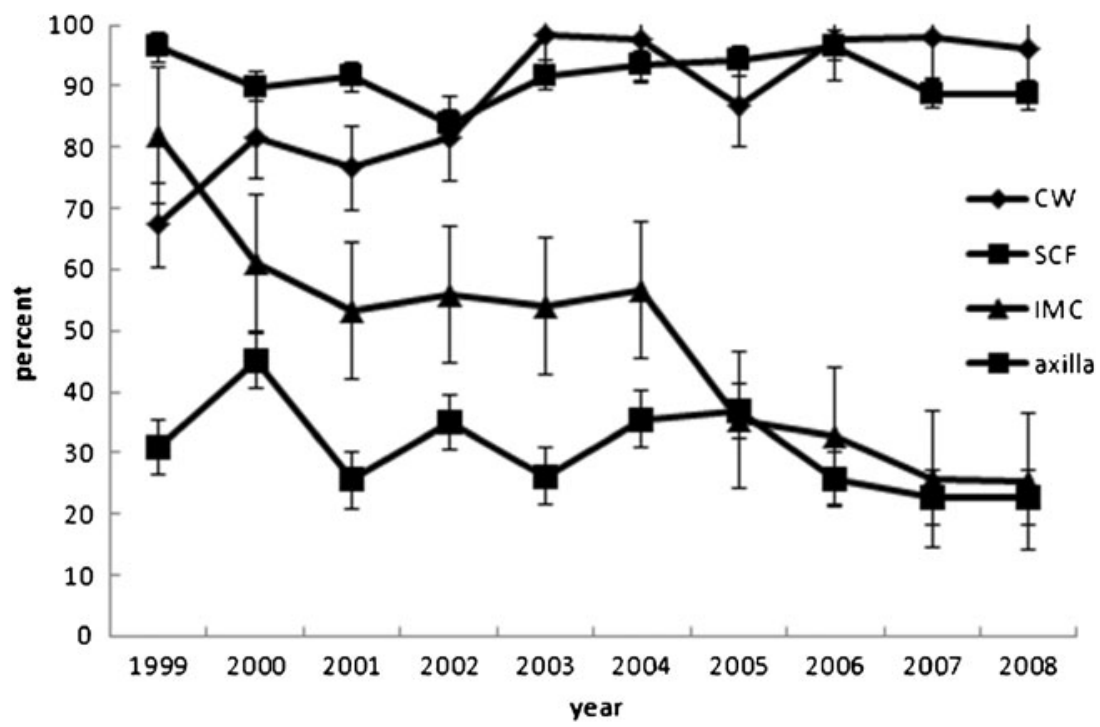

Clinical Oncology recommendation that PMRT includes treatment to the chest wall and the draining lymphatics in the undissected axillary apex and supraclavicular fossa [10]. This finding also demonstrates that the treatment standard in China has improved, mainly because the continuing medical education makes more and more radiation oncologists aware of the evidence abroad and of our own [11].

It is clear that for patients who did not receive PMRT, the chest wall is the most common site of locoregional recurrence, accounting for $55-70 \%$ of all locoregional recurrences [5-8]. In this study, the use of the CW target varied significantly between different regions. Fortunately, from 1999 to 2008, the use of CW RT increased significantly from $67.3 \%$ in 1999 to $96.2 \%$ in 2008, and has remained on a high level since 2003. In CW RT, low-energy electrons are more frequently used, probably due to the thin and less curved chest wall postmastectomy in most Chinese women. Wang et al. has reported a 5-year chest wall recurrence rate of $2.9 \%$ using single low-energy electron beam for postmastectomy chest wall radiation in 328 Chinese women with breast cancer. Figure 4 illustrates typical electron field for chest wall marked on a patient's skin surface [12]. About $20 \%$ more patients in current study received CW RT with photon or cobalt tangential fields. The difference in use of electrons or photon/cobalt tangential fields reflects the availability of different types of machines in different hospitals. The variation in the treatment technique would not be expected to influence the treatment outcome, because Hehr
Fig. 2 Use of locoregional postmastectomy radiotherapy in patients with breast cancer in seven regions

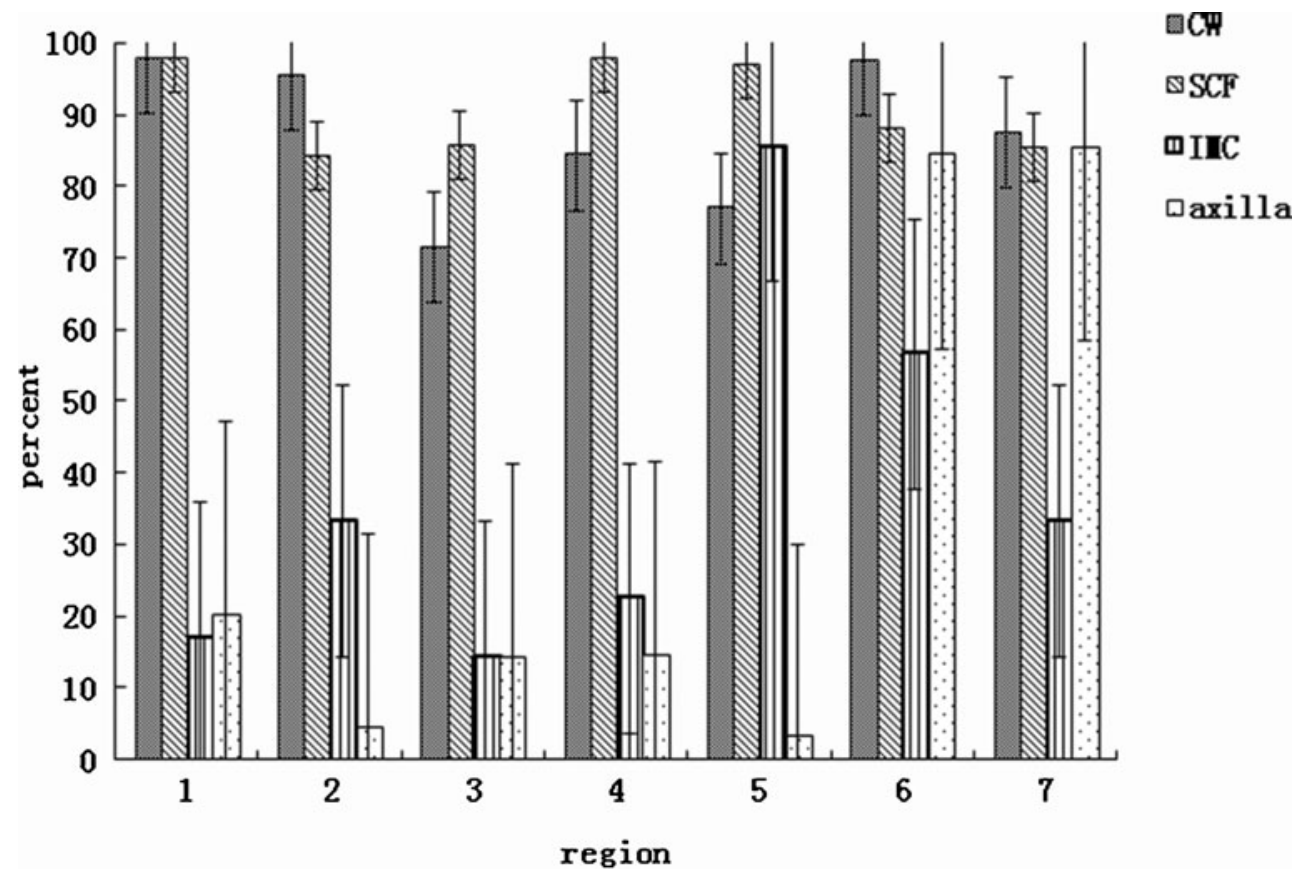


Fig. 3 Distribution of low-, intermediate-, and high-risk patients with breast cancer, from 1999 to 2008

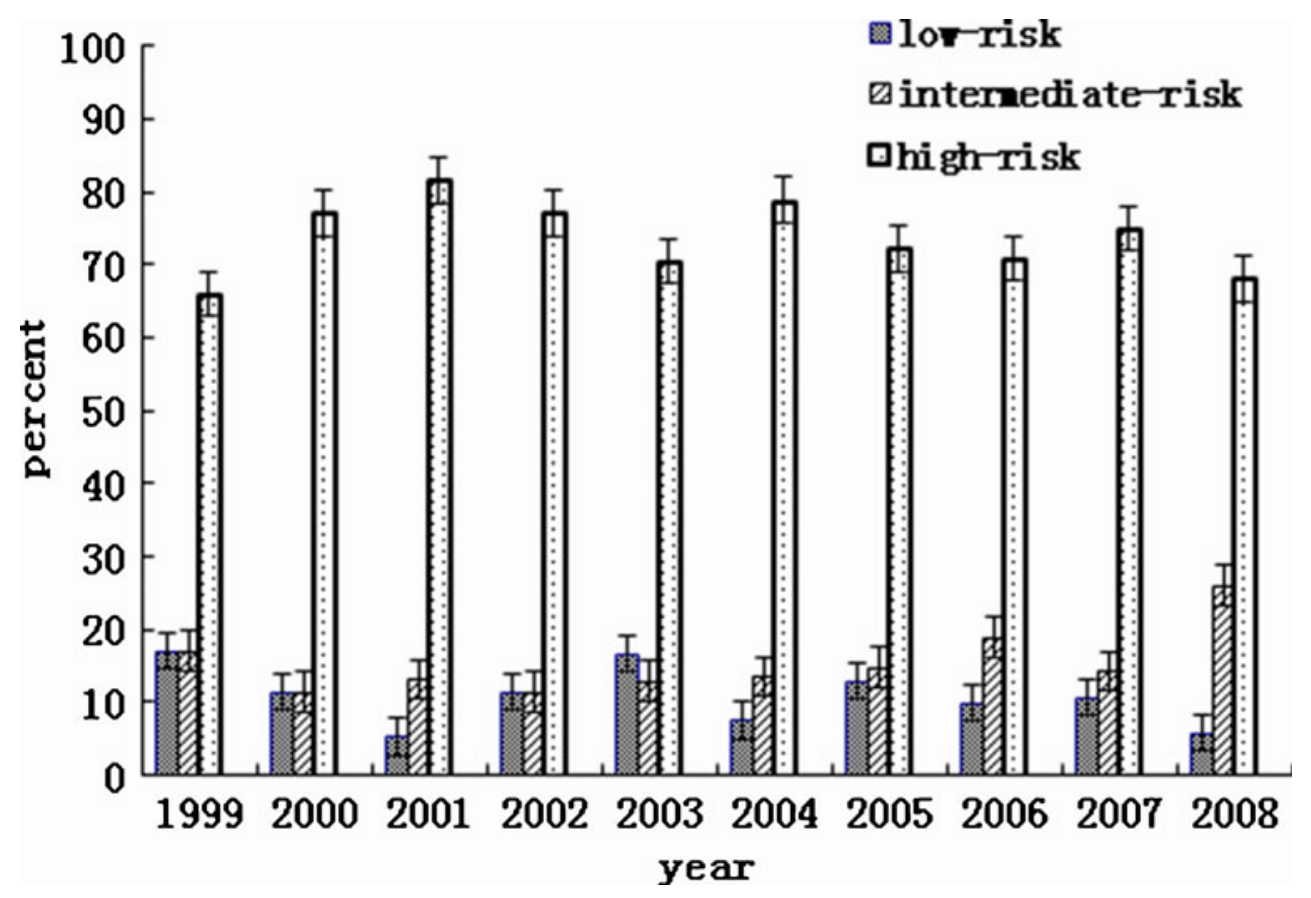

et al. has reported that the 5-year locoregional tumor control rates with electron or photon techniques were comparable, being 92 vs. $89 \%$ [13]. When the chest wall is treated, a tissue equivalent material known as bolus is commonly placed on the skin to reduce the skin-sparing effects of beams and to increase dose to the skin, subcutaneous tissue, and dermal lymphatics. Chest wall bolus was used in about onefourth of patients in this study, though there is little outcome evidence for this bolus technique. Graham et al. reported that parascar bolus and no bolus performed no worse than did whole chest wall bolus with regard to chest wall recurrence. However, daily 1-cm whole chest wall bolus may have an impact on early cessation of radiotherapy caused by skin toxicity, which then may influence chest wall recurrence [14]. An international survey of radiation oncologists found that the use of bolus is frequently determined by the personal preference of the treating radiation oncologist. It was found that $68 \%$ of radiation oncologists use chest wall bolus all the time, $6 \%$ never used bolus, and $26 \%$ used bolus only for specific indications including skin involvement, inflammatory disease, close or positive margins, or lymph-vascular invasionpositive tumors [15]. The guidelines of the American Society of Clinical Oncologists highlighted the lack of evidence to guide clinicians in bolus practice: "Whether it is necessary to apply bolus every day, less frequently, or at all is uncertain" [10]. In regard to this, we typically use bolus during half course of CW RT to increase dose to the dermal lymphatics, meanwhile not to increase the skin toxicity.

The results of a survey conducted in 2004 indicated that the supraclavicular region was the most commonly irrradiated target in mainland China (96.2), followed by IMC (85.2\%), CW (79.0\%), and axilla (74.8\%) [16]. A subsequent survey in 2010 showed that both chest wall and supraclavicular regions were the most common RT target in China $(97.0 \%)$, radiation of IMC and the axilla decreased (39.1 and $50.0 \%$ ) [17]. This change clearly suggested that the CW should be a principal PMRT target for breast cancer, a proposal that achieved a consensus in China.

In the current study, SCF RT was used in more than $80 \%$ of patients, irrespective of year and geographical region. The
Table 4 Use of chest wall and regional node radiation in low-, intermediate-, and high-risk patient groups

$C W$ chest wall, $S C F$ supraclavicular fossa, $I M C$ internal mammary chain, $R T$ radiotherapy

\begin{tabular}{lllll}
\hline & Low-risk & Intermediate-risk & High-risk & $P$ value \\
\hline CW RT & $52(85.2)$ & $78(85.7)$ & $394(94.7)$ & 0.001 \\
No CW RT & $9(14.8)$ & $13(14.3)$ & $22(5.3)$ & \\
SCF RT & $39(63.9)$ & $88(96.7)$ & $399(95.9)$ & $<0.001$ \\
No SCF RT & $22(36.1)$ & $3(3.3)$ & $17(4.1)$ & \\
Axilla RT & $13(21.3)$ & $26(28.6)$ & $130(31.3)$ & 0.274 \\
No axilla RT & $48(78.7)$ & $65(71.4)$ & $286(68.7)$ & \\
IMC RT & $26(42.6)$ & $42(46.2)$ & $180(43.3)$ & 0.868 \\
No IMC RT & $35(57.4)$ & $49(53.8)$ & $236(56.7)$ & \\
\hline
\end{tabular}


Table 5 The correlation between regional node RT field and the axillary nodal status
$S C F$ supraclavicular fossa, $I M C$ internal mammary chain, $R T$ radiotherapy
Number (\%) of patients treated with different regional node RT

$P$ value

Axillary node negative $\quad 1-3$ axillary nodes positive $\quad \geq 4$ axillary nodes positive

\begin{tabular}{lllll}
\hline SCF RT & $89(70.1)$ & $165(98.2)$ & $341(96.3)$ & $<0.001$ \\
No SCF RT & $38(29.9)$ & $3(1.8)$ & $13(3.7)$ & \\
Axilla RT & $25(19.7)$ & $50(29.8)$ & $116(32.8)$ & 0.021 \\
No axilla RT & $102(80.3)$ & $118(70.2)$ & $238(67.2)$ & 0.977 \\
IMC RT & $58(45.7)$ & $76(45.2)$ & $158(44.6)$ & \\
No IMC RT & $69(54.3)$ & $92(54.8)$ & $196(55.4)$ & \\
\hline
\end{tabular}

use of SCF was observed more frequently in patients with positive axillary nodes. Evidence suggests that SCF RT is effective in reducing the rate of SCF failures [2, 4, 18, 19]. The magnitude of benefit is related to the risk of involvement/failure. No surgical resection data exists that has evaluated the risk of SCF involvement at presentation, but it has been found that the risk of SCF failure is closely related to the degree of axillary involvement. A recent study of 1,031 patients who did not receive RT following mastectomy and chemotherapy, found that the 10-year risk of recurrence in the axillary apex/supraclavicular fossa was 14 to $19 \%$ for those with four or more positive lymph nodes, $20 \%$ or greater positive lymph nodes, or extracapsular extension of disease that measured more than $2 \mathrm{~mm}$ [20]. The use of SCF RT increased the risk of brachial plexus neuropathy, radiation pneumonitis, and arm edema. Considering the balance of efficacy and toxicity, SCF RT is recommended for patients with four or more positive axillary nodes. There is controversy about SCF RT in patients with one to three positive nodes [10]. Some physicians believe that SCF RT is not worthwhile as the reported rate of clinical SCF failure is low in this group, whereas others believe that improvements in SCF control may be more readily translated into survival benefit due to the lower risk of distant metastases in patients with one to three positive nodes. In a survey of radiation

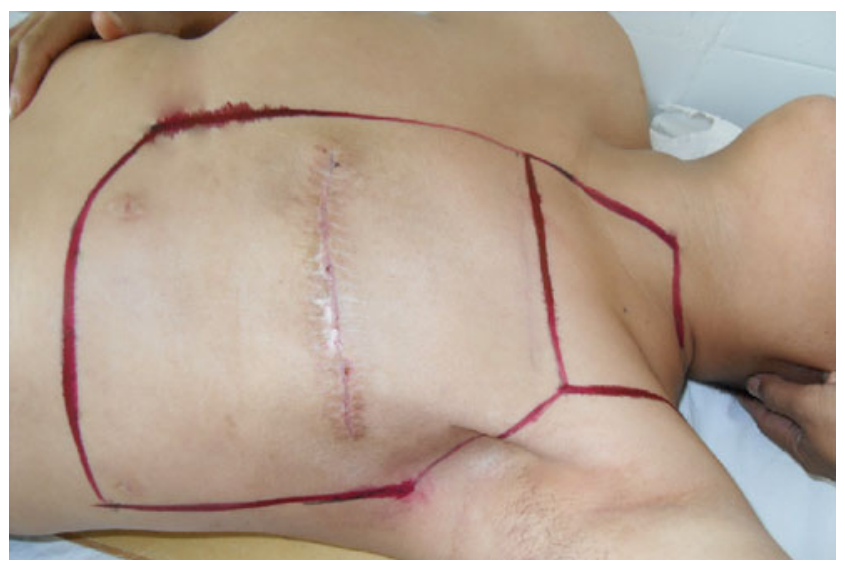

Fig. 4 A typical electron field for chest wall marked on a patient's skin surface oncologists in North America and Europe, respondents were divided on whether to treat the SCF in this setting [21]. Patients with one to three positive nodes have a heterogeneous risk of SCF recurrence and high-risk patients should be identified to deliver SCF radiation. Yates et al. analyzed 1,065 breast cancer patients with one to three positive nodes who did not receive adjuvant SCF radiation, the 10-year SCF recurrence rate was only $9.2 \%$. But higher grade and number of positive lymph nodes were the most significant predictors of SCF recurrence. The 10-year SCF recurrence rate was $30 \%$ in those having grade 3 cancers with three positive nodes [22].

All patients in this study received axillary dissection with a median of 16 lymph nodes recovered, and $29.6 \%$ of patients received axillary RT. The use of axillary RT decreased significantly from 1999 to 2008, and its use varied greatly between different regions. Axillary RT was used more frequently in patients with positive axillary nodes, or with a nodal ratio of $20 \%$ or greater $(p=0.007)$. There is a lack of high-level evidence to support the benefit of post-dissection axillary RT. Controversy exists among American and European radiation oncologists regarding the use of axillary radiotherapy [21]. The 10-year risk of level I or II axillary recurrence was merely $3 \%$ and was not predicted by the extent of axillary disease or extracapsular extension according to a study published by Strom et al., in which all patients had a standard axillary lymph node dissection (median number of lymph nodes recovered was 17) and did not receive RT after treatment with mastectomy and chemotherapy [20]. By contrast, in patients treated in the no RT arms of the Danish PMRT trials and who developed a local-regional recurrence, $43 \%$ experienced the axilla as a component of the recurrence [8]. The higher axillary recurrence rate in these Danish studies was probably due to the less extensive levels I or II dissection (the median number of lymph nodes recovered was seven). This suggests that the decision to include levels I or II as a target for PMRT is largely determined by the completeness of the axillary dissection.

Combined photon and electron, or photon alone were used frequently for SCF RT in this study, electron alone was used in a small number of patients $(6.8 \%)$. Wang et al. has reported that there was no difference in 5-year SCF recurrence rates for patients irradiated with photon, electrons, or combined photon 
and electron $(2.1,3.6$, and $3.2 \%$, respectively) [12]. However, Huang et al. found that patients irradiated with electrons had significantly higher SCF relapse rate than those with photons, which was possibly because the coverage of deep nodes and those located underneath bone would be often insufficient due to inadequate electron depth dose and selective bone absorption [23]. Therefore, we recommend combined photon and electron or photon alone for SCF RT.

The treatment of the IMC in patients undergoing PMRT is controversial and the subject of ongoing phase III studies. In our study, $44.9 \%$ of patients received IMC RT. The use of IMC RT decreased significantly from 1999 to 2008 and it varied greatly between different regions. It was used more frequently in patients with tumors located at the inner quadrant, but was not associated with axillary node status. The justification for including this region is based on previous experience of dissecting the internal mammary chain, which revealed that 35 to $50 \%$ of patients with clinically advanced disease have microscopic involvement of lymph nodes in this area [24, 25]. Randomized trials that have shown a survival advantage for PMRT included the IMC within their treatment target volume $[2,4]$. However, there is a large difference between reported rates of microscopic involvement of the IMC and the subsequent rates of clinically overt failure in the IMC if left untreated. The reported rates of recurrence in the IMC are as low as 0 $1.5 \%[5-7,26]$. Randomized trial shows similar results with or without IMC dissection [27]. There is also a possible risk of long-term cardiac toxicity following IMC RT. A potential compromise approach would be to irradiate the superiorly placed IMC nodal regions, and not the inferiorly placed nodal regions, in order to spare the heart from RT exposure, while at the same time covering the nodal areas more often involved in tumor deposits. In the survey mentioned above, respondents were more likely to use IMC RT in the presence of greater axillary involvement [21]. European respondents were more likely to treat the IMC than their North American counterparts. Academic physicians were more likely to treat the IMC than those in nonacademic positions. Thus, there appears to be significant variations in attitude regarding treatment of the IMC [28]. More evidences are needed to select high-risk patients to deliver IMC RT and patients with positive IMC appear to benefit from IMC radiation. Veronesi et al. reported 663 patients with breast carcinoma mainly in the inner quadrants who underwent a biopsy of IMC. Positive IMC were found in 68 out of 663 cases (10.3\%). Patients with IMC metastases treated with IMC radiation and appropriate systemic treatment achieved an excellent survival (95\% at 5 years) [29].

\section{Conclusion}

Chest wall and supraclavicular fields are the most frequently irradiated targets for PMRT in mainland China. The use of axillary and IMC RT had significantly decreased during the 10 -year period studied. There was a large variation in the use of CW, axillary, and IMC RT between different hospitals, indicating the need for consensus and for evidence.

Acknowledgments This work was supported in part by a grant from Pfizer.

Conflict of interest This study was supported in part by a research grant from Pfizer. Otherwise, Shu-Lian Wang, Ye-xiong Li, Bao-Ning Zhang, Qing-Kun Song, Jing Li, Jin-Hu Fan, Bin Zhang, Hong-Jian Yang, Xiao-Ming Xie, Zhong-Hua Tang, Hui Li, Jia-Yuan Li, Jian-Jun $\mathrm{He}$, You-Lin Qiao claim no conflict of interest. All authors have no financial association with the sponsor of this study.

Ethical standards This study was approved by the institutional review board (IRB) of the Cancer Foundation of China. This survey does not contain any studies with human or animal subjects performed by the any of the authors.

\section{References}

1. Clarke M, Collins R, Darby S, Davies C, Elphinstone P, Evans E, Godwin J, Gray R, Hicks C, James S et al (2005) Effects of radiotherapy and of differences in the extent of surgery for early breast cancer on local recurrence and 15-year survival: an overview of the randomised trials. Lancet 366(9503):2087-2106

2. Overgaard M, Hansen PS, Overgaard J, Rose C, Andersson M, Bach F, Kjaer M, Gadeberg CC, Mouridsen HT, Jensen MB et al (1997) Postoperative radiotherapy in high-risk premenopausal women with breast cancer who receive adjuvant chemotherapy. Danish Breast Cancer Cooperative Group 82b Trial. N Engl J Med 337(14):949-955

3. Ragaz J, Jackson SM, Le N, Plenderleith IH, Spinelli JJ, Basco VE, Wilson KS, Knowling MA, Coppin CM, Paradis M et al (1997) Adjuvant radiotherapy and chemotherapy in node-positive premenopausal women with breast cancer. N Engl J Med 337(14):956-962

4. Overgaard M, Jensen MB, Overgaard J, Hansen PS, Rose C, Andersson M, Kamby C, Kjaer M, Gadeberg CC, Rasmussen $\mathrm{BB}$ et al (1999) Postoperative radiotherapy in high-risk postmenopausal breast-cancer patients given adjuvant tamoxifen: Danish Breast Cancer Cooperative Group DBCG 82c randomised trial. Lancet 353(9165):1641-1648

5. Recht A, Gray R, Davidson NE, Fowble BL, Solin LJ, Cummings FJ, Falkson G, Falkson HC, Taylor SG, Tormey DC (1999) Locoregional failure 10 years after mastectomy and adjuvant chemotherapy with or without tamoxifen without irradiation: experience of the Eastern Cooperative Oncology Group. J clin oncol off J Am Soc Clin Oncol 17(6):1689-1700

6. Katz A, Strom EA, Buchholz TA, Thames HD, Smith CD, Jhingran A, Hortobagyi G, Buzdar AU, Theriault R, Singletary SE et al (2000) Locoregional recurrence patterns after mastectomy and doxorubicinbased chemotherapy: implications for postoperative irradiation. J Clin Oncol Off J Am Soc Clin Oncol 18(15):2817-2827

7. Taghian A, Jeong JH, Mamounas E, Anderson S, Bryant J, Deutsch M, Wolmark N (2004) Patterns of locoregional failure in patients with operable breast cancer treated by mastectomy and adjuvant chemotherapy with or without tamoxifen and without radiotherapy: results from five National Surgical Adjuvant Breast and Bowel Project randomized clinical trials. J Clin Oncol Off J Am Soc Clin Oncol 22(21):4247-4254 
8. Nielsen HM, Overgaard M, Grau C, Jensen AR, Overgaard J (2006) Study of failure pattern among high-risk breast cancer patients with or without postmastectomy radiotherapy in addition to adjuvant systemic therapy: long-term results from the Danish Breast Cancer Cooperative Group DBCG $82 \mathrm{~b}$ and c randomized studies. J Clin Oncol Off J Am Soc Clin Oncol 24(15):2268-2275

9. Li J, Zhang BN, Fan JH, Pang Y, Zhang P, Wang SL, Zheng S, Zhang B, Yang HJ, Xie XM et al (2011) A nation-wide multicenter 10-year (1999-2008) retrospective clinical epidemiological study of female breast cancer in China. BMC cancer 11:364

10. Recht A, Edge SB, Solin LJ, Robinson DS, Estabrook A, Fine RE, Fleming GF, Formenti S, Hudis C, Kirshner JJ et al (2001) Postmastectomy radiotherapy: clinical practice guidelines of the American Society of Clinical Oncology. J Clin Oncol Off J Am Soc Clin Oncol 19(5):1539-1569

11. Shu-Lian Wang Y-XL, Zi-Hao Y (2001) Tagetting in postmastectomy radiotherapy for early stage breast cancer. Chin J Radiat Oncol 10(4):223-227

12. Shu-Lian Wang Y-XL, Yong-Wen S, Wei-Hu W, Jing J, YuePing L, Xin-Fan L, Zi-Hao Y (2012) Postmastectomy chest wall radiotherapy with single low-energy electron beam: an assessment of outcome and prognostic factors. Pract Radiat Oncol 2(2):106-113

13. Hehr T, Classen J, Huth M, Durst I, Christ G, Bamberg M, Budach W et al (2004) Postmastectomy radiotherapy of the chest wall. Comparison of electron-rotation technique and common tangential photon fields. Strahlenther Onkol Organ Dtsch Rontgengesellschaft 180(10):629-636

14. Tieu MT, Graham P, Browne L, Chin YS (2011) The effect of adjuvant postmastectomy radiotherapy bolus technique on local recurrence. Int J Radiat Oncol Biol Phys 81(3):e165-e171

15. Vu TT, Pignol JP, Rakovitch E, Spayne J, Paszat L (2007) Variability in radiation oncologists' opinion on the indication of a bolus in post-mastectomy radiotherapy: an international survey. Clin Oncol (R Coll Radiol) 19(2):115-119

16. Hui Z, Li Y, Yu Z, Liao Z (2006) Survey on use of postmastectomy radiotherapy for breast cancer in China. Int $\mathrm{J}$ Radiat Oncol Biol Phys 66(4):1135-1142

17. Zhou-guang Hui YZ, Jiang-hu Z, Zi-hao Y, Xin-fan L, Jing J, Wei-hu W, Shu-lian W, Yong-wen S, Yue-ping L, Hua R, Hui F, Ye-xiong L (2012) Improvement in postmastectomy radiotherapy for breast cancer in mainland China:comparison of survey in 2010 with 2004. Chin J Radiat Oncol 21(4):352-356

18. Fisher B, Redmond C, Fisher ER, Bauer M, Wolmark N, Wickerham DL, Deutsch M, Montague E, Margolese R, Foster R (1985) Ten-year results of a randomized clinical trial comparing radical mastectomy and total mastectomy with or without radiation. $\mathrm{N}$ Engl J Med 312(11):674-681
19. Ragaz J, Olivotto IA, Spinelli JJ, Phillips N, Jackson SM, Wilson KS, Knowling MA, Coppin CM, Weir L, Gelmon K et al (2005) Locoregional radiation therapy in patients with high-risk breast cancer receiving adjuvant chemotherapy: 20-year results of the British Columbia randomized trial. J Natl Cancer Inst 97(2):116-126

20. Strom EA, Woodward WA, Katz A, Buchholz TA, Perkins GH, Jhingran A, Theriault R, Singletary E, Sahin A, McNeese MD (2005) Clinical investigation: regional nodal failure patterns in breast cancer patients treated with mastectomy without radiotherapy. Int J Radiat Oncol Biol Phys 63(5):1508-1513

21. Ceilley E, Jagsi R, Goldberg S, Grignon L, Kachnic L, Powell S, Taghian A (2005) Radiotherapy for invasive breast cancer in North America and Europe: results of a survey. Int J Radiat Oncol Biol Phys 61(2):365-373

22. Yates L, Kirby A, Crichton S, Gillett C, Cane P, Fentiman I, Sawyer E (2012) Risk factors for regional nodal relapse in breast cancer patients with one to three positive axillary nodes. Int J Radiat Oncol Biol Phys 82(5):2093-2103

23. Huang EY, Chen HC, Sun LM, Fang FM, Hsu HC, Hsiung CY, Huang YJ, Wang CY, Wang CJ (2006) Multivariate analyses of locoregional recurrences and skin complications after postmastectomy radiotherapy using electrons or photons. Int J Radiat Oncol Biol Phys 65(5):1389-1396

24. Huang O, Wang L, Shen K, Lin H, Hu Z, Liu G, Wu J, Lu J, Shao Z, Han Q et al (2008) Breast cancer subpopulation with high risk of internal mammary lymph nodes metastasis: analysis of 2,269 Chinese breast cancer patients treated with extended radical mastectomy. Breast Cancer Res Treat 107(3):379-387

25. Chen RC, Lin NU, Golshan M, Harris JR, Bellon JR (2008) Internal mammary nodes in breast cancer: diagnosis and implications for patient management - a systematic review. J Clin Oncol Off J Am Soc Clin Oncol 26(30):4981-4989

26. Chen L, Gu Y, Leaw S, Wang Z, Wang P, Hu X, Chen J, Lu J, Shao Z (2010) Internal mammary lymph node recurrence: rare but characteristic metastasis site in breast cancer. BMC cancer 10:479

27. Veronesi U, Marubini E, Mariani L, Valagussa P, Zucali R (1999) The dissection of internal mammary nodes does not improve the survival of breast cancer patients. 30-year results of a randomised trial. Eur J Cancer 35(9):1320-1325

28. Taghian A, Jagsi R, Makris A, Goldberg S, Ceilley E, Grignon L, Powell S (2004) Results of a survey regarding irradiation of internal mammary chain in patients with breast cancer: practice is culture driven rather than evidence based. Int J Radiat Oncol Biol Phys 60(3):706-714

29. Veronesi U, Arnone P, Veronesi P, Galimberti V, Luini A, Rotmensz N, Botteri E, Ivaldi GB, Leonardi MC, Viale G et al (2008) The value of radiotherapy on metastatic internal mammary nodes in breast cancer. Results on a large series. Ann Oncol Off J Eur Soc Med Oncol ESMO 19(9):1553-1560 